\title{
LOCAL EXACT CONTROLLABILITY OF THE AGE-DEPENDENT POPULATION DYNAMICS WITH DIFFUSION
}

\author{
BEDR'EDDINE AINSEBA AND SEBASTIAN ANIŢA
}

Received 15 June 2001

We investigate the local exact controllability of a linear age and space population dynamics model where the birth process is nonlocal. The methods we use combine the Carleman estimates for the backward adjoint system, some estimates in the theory of parabolic boundary value problems in $L^{k}$ and the Banach fixed point theorem.

\section{Introduction}

We consider a linear model describing the dynamics of a single species population with age dependence and spatial structure. Let $p(a, t, x)$ be the distribution of individuals of age $a \geq 0$ at time $t \geq 0$ and location $x \in \Omega$, a bounded domain of $\mathbb{R}^{N}, N \in\{1,2,3\}$, with a suitably smooth boundary $\partial \Omega$. Let $a_{\dagger}$ be the life expectancy of an individual and $T$ a positive constant. Let $\beta(a) \geq 0$ be the natural fertility-rate and $\mu(a) \geq 0$ the natural death-rate of individuals of age $a$. We assume that the flux of population takes the form $k \nabla p(a, t, x)$ with $k>0$, where $\nabla$ is the gradient vector with respect to the spatial variable. The evolution of the distribution $p$ is governed by the system

$$
\begin{cases}D p(a, t, x)+\mu(a) p(a, t, x)-k \Delta p(a, t, x)=f(a, x)+m(x) u(a, t, x), & (a, t, x) \in Q_{T}, \\ \frac{\partial p}{\partial v}(a, t, x)=0, & (a, t, x) \in \Sigma_{T}, \\ p(0, t, x)=\int_{0}^{a_{\dagger}} \beta(a) p(a, t, x) d a, & (t, x) \in(0, T) \times \Omega, \\ p(a, 0, x)=p_{0}(a, x), & (a, x) \in\left(0, a_{\dagger}\right) \times \Omega,\end{cases}
$$

where $u$ is a control function, $m$ is the characteristic function of $\omega, f$ is a supply

Copyright (C) 2001 Hindawi Publishing Corporation Abstract and Applied Analysis 6:6 (2001) 357-368

2000 Mathematics Subject Classification: 93B05, 35K05, 46B70, 92D25

URL: http://aaa.hindawi.com/volume-6/S108533750100063X.html 
of individuals and $p_{0}$ is the initial distribution. Here $\omega \subset \Omega$ is a nonempty open subset, $Q_{T}=\left(0, a_{\dagger}\right) \times(0, T) \times \Omega, \Sigma_{T}=\left(0, a_{\dagger}\right) \times(0, T) \times \partial \Omega$.

We have denoted by

$$
D p(a, t, x)=\lim _{\varepsilon \rightarrow 0} \frac{p(a+\varepsilon, t+\varepsilon, x)-p(a, t, x)}{\varepsilon}
$$

the directional derivative of $p$ with respect to the direction $(1,1,0)$. It is obvious that for $p$ smooth enough,

$$
D p=\frac{\partial p}{\partial t}+\frac{\partial p}{\partial a} .
$$

Let $p_{s}$ be a steady-state of (1.1), corresponding to $u \equiv 0$ and such that

$$
p_{s}(a, x) \geq \rho_{0}>0 \quad \text { a.e. }(a, x) \in\left(0, a_{1}\right) \times \Omega,
$$

where $\rho_{0}>0$ is constant and $a_{1}$ is a constant which will be later defined and belongs to $\left(0, a_{\dagger}\right)$.

The main goal of this paper is to prove the existence of a control $u$ such that the solution of (1.1) satisfies

$$
\begin{array}{ll}
p(a, T, x)=p_{s}(a, x) & \text { a.e. }(a, x) \in\left(0, a_{\dagger}\right) \times \Omega, \\
p(a, t, x) \geq 0 & \text { a.e. }(a, t, x) \in Q_{T} .
\end{array}
$$

Condition (1.6) is natural because $p$ represents the density of a population.

We notice that if $p$ is the solution to (1.1), then $p-p_{s}$ is the solution to

$$
\begin{cases}D p+\mu(a) p-k \Delta p=m(x) u(a, t, x), & (a, t, x) \in Q_{T}, \\ \frac{\partial p}{\partial v}(a, t, x)=0, & (a, t, x) \in \Sigma_{T}, \\ p(0, t, x)=\int_{0}^{a_{\dagger}} \beta(a) p(a, t, x) d a, & (t, x) \in(0, T) \times \Omega, \\ p(a, 0, x)=\bar{p}_{0}(a, x), & (a, x) \in\left(0, a_{\dagger}\right) \times \Omega,\end{cases}
$$

where $\bar{p}_{0}=p_{0}-p_{s}$.

The above formulated problem is equivalent with the exact null controllability problem for (1.7). If we denote now by $p$ the solution to (1.7), then condition (1.6) becomes

$$
p(a, t, x) \geq-p_{s}(a, x) \quad \text { a.e. }(a, t, x) \in Q_{T} .
$$

The main result of this paper amounts to saying that system (1.7) is exactly null controllable for $p_{0}$ in a neighborhood of $p_{s}$.

We recall that the internal null controllability of the linear heat equation, when the control acts on a subset of the domain, was established by Lebeau and Robbiano [10] and was later extended to some semilinear equation by Fursikov 
and Imanuvilov [4], in the sublinear case and by Barbu [2] and Fernandez-Cara [3], in the superlinear case.

The paper is organized as follows. We first give the hypotheses and state the main result. The existence of a steady-state of (1.1) with $u \equiv 0$ is established in Section 3. The proof of the local exact null controllability is given in Section 4. The proof is based on Carleman's inequality for the backward adjoint system associated with (1.7).

\section{Assumptions and the main result}

Assume that the following hypotheses hold:

(A1) $\beta \in L^{\infty}\left(0, a_{\dagger}\right), \beta(a) \geq 0$ a.e. $a \in\left(0, a_{\dagger}\right)$,

(A2) there exists $a_{0}, a_{1} \in\left(0, a_{\dagger}\right)$ such that $\beta(a)=0$ a.e. $a \in\left(0, a_{0}\right) \cup\left(a_{1}, a_{\dagger}\right)$, $\beta(a)>0$ a.e. in $\left(a_{0}, a_{1}\right)$,

(A3) $\mu \in L_{\text {loc }}^{1}\left(\left[0, a_{\dagger}\right)\right), \mu(a) \geq 0$ a.e. $a \in\left(0, a_{\dagger}\right)$,

(A4) $\int_{0}^{a_{\dagger}} \mu(a) d a=+\infty$,

(A5) $p_{0} \in L^{\infty}\left(\left(0, a_{\dagger}\right) \times \Omega\right), p_{0}(a, t) \geq 0$ a.e. in $\left(0, a_{\dagger}\right) \times \Omega, f \in L^{\infty}\left(\left(0, a_{\dagger}\right)\right.$ $\times \Omega), f(a, x) \geq 0$ a.e. in $\left(0, a_{\dagger}\right) \times \Omega$.

For the biological significance of the hypotheses and the basic existence results for the solution to (1.1) we refer to $[5,6,11]$.

Let $p_{s}$ be a steady-state of (1.1), corresponding to $u \equiv 0$ and such that

$$
p_{s}(a, x) \geq \rho_{0}>0 \quad \text { a.e. }(a, x) \in\left(0, a_{1}\right) \times \Omega,
$$

where $\rho_{0}>0$ is constant.

Denote by $\bar{p}_{0}=p_{0}-p_{s}$. Then we have the following theorem.

THEOREM 2.1. If $\left\|\bar{p}_{0}\right\|_{L^{\infty}\left(\left(0, a_{\dagger}\right) \times \Omega\right)}$ is small enough, then there exists $u \in L^{2}\left(Q_{T}\right)$ such that the solution $p$ of (1.7) satisfies

$$
\begin{gathered}
p(a, T, x)=0 \quad \text { a.e. }(a, x) \in\left(0, a_{\dagger}\right) \times \Omega, \\
p(a, t, x) \geq-p_{s}(a, x) \quad \text { a.e. }(a, t, x) \in Q_{T} .
\end{gathered}
$$

\section{Existence of steady states to (1.1)}

In this section, we will study the existence of $p_{s}$, a steady-state of (1.1), corresponding to $u \equiv 0$, which satisfies (1.4). The steady-state $p_{s}$ should be a solution to

$$
\begin{cases}\frac{\partial p_{s}}{\partial a}+\mu(a) p_{s}-k \Delta p_{s}=f(a, x), & (a, x) \in\left(0, a_{\dagger}\right) \times \Omega, \\ \frac{\partial p_{s}}{\partial v}(a, x)=0, & (a, x) \in\left(0, a_{\dagger}\right) \times \partial \Omega, \\ p_{s}(0, x)=\int_{0}^{a_{\dagger}} \beta(a) p_{s}(a, x) d a, & x \in \Omega .\end{cases}
$$


Denote by $R=\int_{0}^{a_{\dagger}} \beta(a) e^{-\int_{0}^{a} \mu(s) d s} d a$ the reproductive number and by $f_{0}$ a nonnegative constant.

THEOREM 3.1. If $R<1$ and $f(a, x) \geq f_{0}>0$ a.e. $(a, x) \in\left(0, a_{\dagger}\right) \times \Omega$, then there exists a unique solution to (3.1), which in addition satisfies (1.4).

If $R=1$ and $f \equiv 0$, then there exist infinitely many solutions to (3.1), which satisfy (1.4). If $R>1$, then there is no nonnegative solution to (3.1), satisfying (1.4).

Proof. If $R<1$, then there exists a unique (and nonnegative) solution to (3.1) via Banach fixed point theorem. If, in addition, $f(a, x) \geq f_{0}>0$ a.e. $(a, x) \in$ $\left(0, a_{\dagger}\right) \times \Omega$, then by the comparison result in [5] we get that

$$
p_{s}(a, x) \geq p_{i}(a, t, x) \quad \text { a.e. }(a, t, x) \in Q=\left(0, a_{\dagger}\right) \times(0,+\infty) \times \Omega,
$$

where $p_{i}$ is the solution to

$$
\begin{array}{ll}
D p_{i}+\mu p_{i}-k \Delta p_{i}=f_{0}, & (a, t, x) \in Q, \\
\frac{\partial p_{i}}{\partial v}=0, & (a, t, x) \in \Sigma, \\
p_{i}(0, t, x)=\int_{0}^{a_{\dagger}} \beta(a) p_{i}(a, t, x) d a, & (t, x) \in(0,+\infty) \times \Omega, \\
p_{i}(a, 0, x)=0, & (a, x) \in\left(0, a_{\dagger}\right) \times \Omega
\end{array}
$$

$\left(\Sigma=\left(0, a_{\dagger}\right) \times(0,+\infty) \times \partial \Omega\right) ; p_{i}$ does not explicitly depend on $x$. So, we will write $p_{i}(a, t)$ instead of $p_{i}(a, t, x)$ and

$$
p_{s}(a, x) \geq p_{i}(a, t) \quad \forall t \in[0,+\infty), \quad \text { a.e. }(a, x) \in\left(0, a_{\dagger}\right) \times \Omega,
$$

where $p_{i}$ is the solution of

$$
\begin{array}{ll}
D p_{i}+\mu p_{i}=f_{0}, & (a, t) \in\left(0, a_{\dagger}\right) \times(0,+\infty), \\
p_{i}(0, t)=\int_{0}^{a_{\dagger}} \beta(a) p_{i}(a, t) d a, & t \in(0,+\infty), \\
p_{i}(a, 0)=0, & a \in\left(0, a_{\dagger}\right) .
\end{array}
$$

For $t>a_{\dagger}$ we have

$$
p_{i}(0, t)>0, \quad p_{i}(0, t) \text { is continuous with respect to } t
$$

(see [7]). As a consequence we obtain that there exists $\rho_{0}>0$ such that, for $t$ large enough, and for any $a \in\left(0, a_{1}\right)$,

$$
p_{i}(a, t)>\rho_{0},
$$

and in conclusion we get that $p_{s}$ satisfies (1.4). 
If $R=1$ and $f \equiv 0$, then any function defined by

$$
p(a, x)=c e^{-\int_{0}^{a} \mu(s) d s} d a, \quad(a, x) \in\left(0, a_{\dagger}\right) \times \Omega
$$

is a solution to (3.1) (for any $c \in \mathbb{R}$ ). In fact these are all the solutions to (3.1) in this case. It is now obvious that there exist infinitely many solutions to (3.1), which satisfy (1.4).

If $R>1$ and if it would exist a nonnegative solution $p_{s}$ to (3.1) satisfying (1.4), then $p(a, t, x)=p_{s}(a, x),(a, t, x) \in Q$ is the solution to

$$
\begin{array}{ll}
D p+\mu p-k \Delta p=f(a, x), & (a, t, x) \in Q, \\
\frac{\partial p}{\partial v}=0, & (a, t, x) \in \Sigma, \\
p(0, t, x)=\int_{0}^{a_{\dagger}} \beta(a) p(a, t, x), & (t, x) \in(0,+\infty) \times \Omega, \\
p(a, 0, x)=p_{s}(a, x), & (a, x) \in\left(0, a_{\dagger}\right) \times \Omega
\end{array}
$$

and for $t \rightarrow+\infty$ we have (see [9])

$$
\lim _{t \rightarrow+\infty}\|p(t)\|_{L^{2}\left(\left(0, a_{\dagger}\right) \times \Omega\right)}=+\infty .
$$

On the other hand,

$$
\|p(t)\|_{L^{2}\left(\left(0, a_{\dagger}\right) \times \Omega\right)}=\left\|p_{s}\right\|_{L^{2}\left(\left(0, a_{\dagger}\right) \times \Omega\right)},
$$

and so $\left\|p_{s}\right\|_{L^{2}\left(\left(0, a_{\dagger}\right) \times \Omega\right)}=+\infty$, which is absurd.

\section{Proof of Theorem 2.1}

In what follows we will use the general Carleman inequality for linear parabolic equations given in [4]. Namely, let $\tilde{\omega} \subset \subset \omega$ be a nonempty bounded set, $T_{0} \in$ $(0,+\infty)$ and $\psi \in C^{2}(\bar{\Omega})$ be such that

$$
\begin{aligned}
\psi(x)>0, & \forall x \in \Omega, \\
\psi(x)=0, & \forall x \in \partial \Omega, \\
|\nabla \psi(x)|>0, & \forall x \in \bar{\Omega} \backslash \tilde{\omega}
\end{aligned}
$$

and set

$$
\alpha(t, x)=\frac{e^{\lambda \psi(x)}-e^{2 \lambda\|\psi\|_{C(\bar{\Omega})}}}{t\left(T_{0}-t\right)},
$$

where $\lambda$ is an appropriate positive constant.

Denote by $D_{T_{0}}=\left(0, T_{0}\right) \times \Omega$. 
362 Controllability of the population dynamics

Lemma 4.1. There exist positive constants $C_{1}, s_{1}$ such that

$$
\begin{aligned}
& \frac{1}{s} \int_{D_{T_{0}}} t\left(T_{0}-t\right) e^{2 s \alpha}\left(\left|w_{t}\right|^{2}+|\Delta w|^{2}\right) d x d t+s \int_{D_{T_{0}}} \frac{e^{2 s \alpha}}{t\left(T_{0}-t\right)}|\nabla w|^{2} d x d t \\
& \quad+s^{3} \int_{D_{T_{0}}} \frac{e^{2 s \alpha}}{t^{3}\left(T_{0}-t\right)^{3}}|w|^{2} d x d t \\
& \leq C_{1}\left[\int_{D_{T_{0}}} e^{2 s \alpha}\left|w_{t}+\Delta w\right|^{2} d x d t+s^{3} \int_{\left(0, T_{0}\right) \times \omega} \frac{e^{2 s \alpha}}{t^{3}\left(T_{0}-t\right)^{3}}|w|^{2} d x d t\right],
\end{aligned}
$$

for all $w \in C^{2}\left(\bar{D}_{T_{0}}\right),(\partial w / \partial v)(t, x)=0, \forall(t, x) \in\left(0, T_{0}\right) \times \partial \Omega$ and $s \geq s_{1}$.

For the proof of this result we refer to [4]. Let $T_{0} \in\left(0, \min \left\{a_{0}, T / 2, a_{\dagger}-a_{1}\right\}\right)$. Define

$$
K=L^{\infty}\left(\left(0,2 T_{0}\right) \times \Omega\right)
$$

In what follows we will denote by the same symbol $C$, several constants independent of $\bar{p}_{0}$ and all other variables.

For $b \in K$ arbitrary but fixed and for any $\varepsilon>0$, consider the following optimal control problem:

$$
\operatorname{Minimize}\left\{\int_{G} \int_{\Omega} \varphi(a, t, x)|u(a, t, x)|^{2} d x d t d a+\frac{1}{\varepsilon} \int_{\Gamma_{0}} \int_{\Omega}|p(a, t, x)|^{2} d x d l\right\} \text {, }
$$

subject to (4.7) ( $u \in L^{2}\left(Q_{2 T_{0}}\right)$ and $p$ is the solution of (4.7) corresponding to $\left.u\right)$. Here

$$
G=\left(0, a_{\dagger}\right) \times\left(0, T_{0}\right) \cup\left(0, T_{0}\right) \times\left(T_{0}, 2 T_{0}\right)
$$

(see Figure 4.1)

$$
\begin{gathered}
\Gamma_{0}=\left\{T_{0}\right\} \times\left(T_{0}, 2 T_{0}\right) \cup\left(T_{0}, a_{\dagger}-T_{0}\right) \times\left\{T_{0}\right\}, \\
\varphi(a, t, x)= \begin{cases}e^{-2 s \alpha(t, x)} t^{3}\left(T_{0}-t\right)^{3}, & \text { if } t<a,(a, t) \in G, \\
e^{-2 s \alpha(a, x)} a^{3}\left(T_{0}-a\right)^{3}, & \text { if } a<t,(a, t) \in G,\end{cases}
\end{gathered}
$$


B. Ainseba and S. Aniţa 363

$$
\begin{array}{ll}
D p+\mu p-k \Delta p=m(x) \tilde{m}(a, t) u(a, t, x), & (a, t, x) \in Q_{2 T_{0},} \\
\frac{\partial p}{\partial \nu}=0, & (a, t, x) \in \Sigma_{2 T_{0}}, \\
p(0, t, x)=b(t, x), & (t, x) \in\left(0,2 T_{0}\right) \times \Omega, \\
p(a, 0, x)=p_{0}(a, x), & (a, x) \in\left(0, a_{\dagger}\right) \times \Omega .
\end{array}
$$

Here $\tilde{m}$ is the characteristic function of $G$.

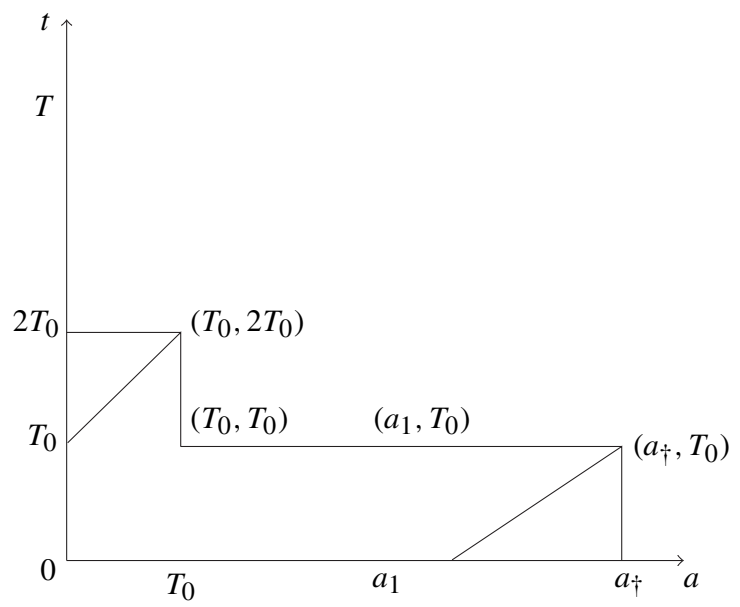

Figure 4.1. Case where $T_{0}=a_{0}$.

Denote by $\Psi_{\varepsilon}(u)$ the value of the cost function in $u$. Since the cost function $\Psi_{\varepsilon}: L^{2}\left(Q_{2 T_{0}}\right) \rightarrow \mathbb{R}^{+}$is convex, continuous and

$$
\lim _{\|u\|_{L^{2}\left(Q_{2 T_{0}}\right)} \rightarrow+\infty} \Psi_{\varepsilon}(u)=+\infty,
$$

then it follows that there exists at least one minimum point for $\Psi_{\varepsilon}$ and consequently an optimal pair $\left(u_{\varepsilon}, p_{\varepsilon}\right)$ for (4.5). By standard arguments we have

$$
u_{\varepsilon}(a, t, x)=m(x) \tilde{m}(a, t) q_{\varepsilon}(a, t, x) \varphi^{-1}(a, t, x) \quad \text { a.e. }(a, t, x) \in Q_{2 T_{0}},
$$

where $q_{\varepsilon}$ is the solution of

$$
\begin{array}{ll}
D q-\mu q+k \Delta q=0, & (a, t, x) \in G \times \Omega, \\
\frac{\partial q}{\partial v}=0, & (a, t, x) \in G \times \partial \Omega, \\
q(a, t, x)=0, & (a, t, x) \in\left(\Gamma \backslash \Gamma_{0}\right) \times \Omega, \\
q(a, t, x)=-\frac{1}{\varepsilon} p_{\varepsilon}(a, t, x), & (a, t, x) \in \Gamma_{0} \times \Omega .
\end{array}
$$

Here $\Gamma=\left(0, T_{0}\right) \times\left\{2 T_{0}\right\} \cup\left\{a_{\dagger}\right\} \times\left(0, T_{0}\right) \cup \Gamma_{0} \cup\left(a_{\dagger}-T_{0}, a_{\dagger}\right) \times\left\{T_{0}\right\}$. 
Multiplying the first equation in (4.10) by $p_{\varepsilon}$ and integrating on $Q_{2 T_{0}}$, we obtain after some calculation (and using (4.7) and (4.9)) that

$$
\begin{aligned}
\int_{G} \int_{\omega} \varphi(a, t, x)\left|u_{\varepsilon}(a, t, x)\right|^{2} d x d a d t+\frac{1}{\varepsilon} \int_{\Gamma_{0}} \int_{\Omega}\left|p_{\epsilon}(a, t, x)\right|^{2} d x d l \\
=-\int_{0}^{T_{0}} \int_{\Omega} b(t, x) q_{\varepsilon}(0, t, x) d x d t \\
-\int_{0}^{a_{\dagger}-T_{0}} \int_{\Omega} \bar{p}_{0}(a, x) q_{\varepsilon}(a, 0, x) d x d a
\end{aligned}
$$

Let $S$ be an arbitrary characteristic line of equation

$$
S=\left\{(\gamma+t, \theta+t) ; t \in\left(0, T_{0}\right)\right\}, \quad(\gamma, \theta) \in\left(0, a_{\dagger}-T_{0}\right) \times\{0\} \cup\{0\} \times\left(0, T_{0}\right) .
$$

Define

$$
\begin{aligned}
\tilde{u}(t, x) & =u(\gamma+t, \theta+t, x), & & (t, x) \in\left(0, T_{0}\right) \times \Omega, \\
\tilde{p}_{\varepsilon}(t, x) & =p_{\varepsilon}(\gamma+t, \theta+t, x), & & (t, x) \in\left(0, T_{0}\right) \times \Omega, \\
\tilde{q}_{\varepsilon}(t, x) & =q_{\varepsilon}(\gamma+t, \theta+t, x), & & (t, x) \in\left(0, T_{0}\right) \times \Omega, \\
\tilde{\mu}(t) & =\mu(\gamma+t), & & t \in\left(0, T_{0}\right) .
\end{aligned}
$$

$\left(\tilde{u}_{\varepsilon}, \tilde{p}_{\varepsilon}, \tilde{q}_{\varepsilon}\right)$ satisfies

$$
\begin{aligned}
&\left(\tilde{p}_{\varepsilon}\right)_{t}+\tilde{\mu} \tilde{p}_{\varepsilon}-k \Delta \tilde{p}_{\varepsilon}=m(x) \tilde{u}_{\varepsilon}(t, x),(t, x) \in\left(0, T_{0}\right) \times \Omega, \\
& \frac{\partial \tilde{p}_{\varepsilon}}{\partial v}=0,(t, x) \in\left(0, T_{0}\right) \times \partial \Omega, \\
& \tilde{p}_{\varepsilon}(0, x)=\left\{\begin{array}{ll}
b(\theta, x), & \gamma=0 \\
\bar{p}_{0}(\gamma, x), & \theta=0
\end{array} \quad x \in \Omega .\right.
\end{aligned}
$$

This yields

$$
\tilde{u}_{\varepsilon}(t, x)=m(x) \tilde{q}_{\varepsilon}(t, x) \cdot \frac{e^{2 s \alpha(t, x)}}{t^{3}\left(T_{0}-t\right)^{3}}
$$

a.e. $(t, x) \in\left(0, T_{0}\right) \times \Omega$,

$$
\begin{array}{ll}
\left(\tilde{q}_{\varepsilon}\right)_{t}+k \Delta \tilde{q}_{\varepsilon}=\tilde{\mu} \tilde{q}_{\varepsilon}, & (t, x) \in\left(0, T_{0}\right) \times \Omega, \\
\frac{\partial \tilde{q}_{\varepsilon}}{\partial v}=0, & (t, x) \in\left(0, T_{0}\right) \times \partial \Omega, \\
\tilde{q}_{\varepsilon}\left(T_{0}, x\right)=-\frac{1}{\varepsilon} \tilde{p}_{\varepsilon}\left(T_{0}, x\right) & x \in \Omega .
\end{array}
$$


Multiplying the first equation in (4.16) by $\tilde{p}_{\varepsilon}$ and integrating on $D_{T_{0}}$, we obtain that

$$
\begin{gathered}
\int_{0}^{T_{0}} \int_{\omega} e^{-2 s \alpha(t, x)} t^{3}\left(T_{0}-t\right)^{3}\left|\tilde{u}_{\varepsilon}(t, x)\right|^{2} d x d t+\frac{1}{\varepsilon} \int_{\Omega}\left|\tilde{p}_{\varepsilon}\left(T_{0}, x\right)\right|^{2} d x \\
=-\int_{\Omega} \tilde{p}_{\varepsilon}(0, x) \tilde{q}_{\varepsilon}(0, x) d x .
\end{gathered}
$$

By Carleman's inequality (4.3) we infer that

$$
\begin{aligned}
& \int_{0}^{T_{0}} \int_{\Omega} e^{2 s \alpha} {\left[\frac{t\left(T_{0}-t\right)}{s}\left(\left|\left(\tilde{q}_{\varepsilon}\right)_{t}\right|^{2}+\left|\Delta \tilde{q}_{\varepsilon}\right|^{2}\right)\right.} \\
&\left.+\frac{s}{t\left(T_{0}-t\right)}\left|\nabla \tilde{q}_{\varepsilon}\right|^{2}+\frac{s^{3}}{t^{3}\left(T_{0}-t\right)^{3}}\left|\tilde{q}_{\varepsilon}\right|^{2}\right] d x d t \\
& \leq C_{1}\left[\int_{0}^{T_{0}} \int_{\Omega} e^{2 s \alpha}\|\tilde{\mu}\|_{C\left(\left[0, T_{0}\right]\right)}^{2} \cdot\left|\tilde{q}_{\varepsilon}\right|^{2} d x d t\right. \\
&\left.+s^{3} \int_{\left(0, T_{0}\right) \times \omega} \frac{e^{2 s \alpha}}{t^{3}\left(T_{0}-t\right)^{3}}\left|\tilde{q}_{\varepsilon}\right|^{2} d x d t\right]
\end{aligned}
$$

and consequently

$$
\begin{aligned}
\int_{0}^{T_{0}} \int_{\Omega} e^{2 s \alpha}[ & \frac{t\left(T_{0}-t\right)}{s}\left(\left|\left(\tilde{q}_{\varepsilon}\right)_{t}\right|^{2}+\left|\Delta \tilde{q}_{\varepsilon}\right|^{2}\right) \\
& \left.+\frac{s}{t\left(T_{0}-t\right)}\left|\nabla \tilde{q}_{\varepsilon}\right|^{2}+\frac{s^{3}}{t^{3}\left(T_{0}-t\right)^{3}}\left|\tilde{q}_{\varepsilon}\right|^{2}\right] d x d t \\
\leq C & \int_{0}^{T_{0}} \int_{\omega} e^{2 s \alpha} \frac{s^{3}}{t^{3}\left(T_{0}-t\right)^{3}}\left|\tilde{q}_{\varepsilon}\right|^{2} d x d t
\end{aligned}
$$

for $s \geq \max \left(s_{1}, C\|\mu\|_{C\left(\left[0, a_{\dagger}-T_{0}\right]\right)}^{2 / 3}\right)$.

Multiplying the first equation in (4.16) by $\tilde{q}_{\varepsilon}$ we obtain that

$$
\begin{gathered}
\frac{1}{2} \frac{d}{d t} \int_{\Omega}\left|\tilde{q}_{\varepsilon}(t, x)\right|^{2} d x-k \int_{\Omega}\left|\nabla \tilde{q}_{\varepsilon}(t, x)\right|^{2} d x-\int_{\Omega} \tilde{\mu}(t)\left|\tilde{q}_{\varepsilon}(t, x)\right|^{2} d x=0 \\
\frac{d}{d t} \int_{\Omega}\left|\tilde{q}_{\varepsilon}(t, x)\right|^{2} d x \geq 0 \quad \text { a.e. } t \in\left(0, T_{0}\right)
\end{gathered}
$$

Integrating the last inequality we get that

$$
\int_{\Omega}\left|\tilde{q}_{\varepsilon}(0, x)\right|^{2} d x \leq C \int_{0}^{T_{0}} \int_{\Omega}\left|\tilde{q}_{\varepsilon}(t, x)\right|^{2} \frac{e^{2 s \alpha(x, t)}}{t^{3}\left(T_{0}-t\right)^{3}} d x
$$

and by Carleman's inequality we have

$$
\int_{\Omega}\left|\tilde{q}_{\varepsilon}(0, x)\right|^{2} d x \leq C \int_{0}^{T_{0}} \int_{\omega}\left|\tilde{q}_{\varepsilon}(t, x)\right|^{2} \cdot \frac{e^{2 s \alpha(x, t)}}{t^{3}\left(T_{0}-t\right)^{3}} d x d t .
$$


By Young's inequality (4.16), (4.22), and (4.15) we obtain

$$
\begin{aligned}
& \int_{\left(0, T_{0}\right) \times \omega} e^{-2 s \alpha} t^{3}\left(T_{0}-t\right)^{3}\left|\tilde{u}_{\varepsilon}(t, x)\right|^{2} d x d t+\frac{1}{\varepsilon} \int_{\Omega}\left|\tilde{p}_{\varepsilon}\left(T_{0}, x\right)\right|^{2} d x \\
& \quad \leq C\left\|\tilde{p}_{\varepsilon}(0)\right\|_{L^{2}(\Omega)}^{2},
\end{aligned}
$$

for $s \geq \max \left(s_{1}, C\|\mu\|_{C\left(\left[0, a_{\dagger}-T_{0}\right]\right)}^{2 / 3}\right)$.

Using now (4.19) we get

$$
\begin{gathered}
\int_{0}^{T_{0}} \int_{\Omega} e^{2 s \alpha}\left[\frac{t\left(T_{0}-t\right)}{s}\left(\left|\left(\tilde{q}_{\varepsilon}\right)_{t}\right|^{2}+\left|\Delta \tilde{q}_{\varepsilon}\right|^{2}\right)+\frac{s}{t\left(T_{0}-t\right)}\left|\nabla \tilde{q}_{\varepsilon}\right|^{2}\right. \\
\left.\quad+\frac{s^{3}}{t^{3}\left(T_{0}-t\right)^{3}}\left|\tilde{q}_{\varepsilon}\right|^{2}\right] d x d t \\
\leq C\left\|\tilde{p}_{\varepsilon}(0)\right\|_{L^{2}(\Omega)}^{2},
\end{gathered}
$$

for any $\varepsilon>0$ and consequently

$$
\left\|\tilde{v}_{\varepsilon}\right\|_{W_{2}^{1,2}\left(\left(0, T_{0}\right) \times \Omega\right)}^{2} \leq C\left\|\tilde{p}_{\varepsilon}(0)\right\|_{L^{2}(\Omega)}^{2},
$$

where $\tilde{v}_{\varepsilon}(t, x)=\left(e^{2 s \alpha(t, x)} / t^{3}\left(T_{0}-t\right)^{3}\right) \tilde{q}_{\varepsilon},(t, x) \in\left(0, T_{0}\right) \times \Omega$. As

$$
W_{2}^{1,2}\left(\left(0, T_{0}\right) \times \Omega\right) \subset L^{l}\left(\left(0, T_{0}\right) \times \Omega\right)
$$

(where $l=+\infty$ for $N=1,2$ and $l=10$ for $N=3$ ), we may infer that

$$
\left\|\tilde{u}_{\varepsilon}\right\|_{L^{10}\left(\left(0, T_{0}\right) \times \Omega\right)}^{2}=\left\|m \tilde{v}_{\varepsilon}\right\|_{L^{10}\left(\left(0, T_{0}\right) \times \Omega\right)}^{2} \leq C\left\|\tilde{p}_{\varepsilon}(0)\right\|_{L^{2}(\Omega)}^{2},
$$

for any $\varepsilon>0$ and $s \geq \max \left(s_{1}, C\|\mu\|_{C\left(\left[0, a_{\dagger}-T_{0}\right]\right)}^{2 / 3}\right)$.

The last estimate and the existence theory of parabolic boundary value problems in $L^{r}$ (see [8]) imply that on a subsequence we have that

$$
\begin{aligned}
& \tilde{u}_{\varepsilon} \longrightarrow \tilde{u} \quad \text { weakly in } L^{10}\left(\left(0, T_{0}\right) \times \Omega\right) \\
& \tilde{p}_{\varepsilon} \longrightarrow \tilde{p}^{\tilde{u}} \quad \text { weakly in } W_{10}^{1,2}\left(\left(0, T_{0}\right) \times \Omega\right),
\end{aligned}
$$

where $\left(\tilde{u}, \tilde{p}^{\tilde{u}}\right)$ satisfies (4.14) and

$$
\tilde{p}^{\tilde{u}}\left(T_{0}, x\right)=0 \quad \text { a.e. } x \in \Omega \text {. }
$$

By (4.14) we get

$$
\left\|\tilde{p}^{\tilde{u}}\right\|_{L^{\infty}\left(\left(0, T_{0}\right) \times \Omega\right)}^{2} \leq C\left(\left\|\tilde{p}_{\varepsilon}(0)\right\|_{L^{\infty}(\Omega)}^{2}+\left\|m \tilde{u}_{\varepsilon}\right\|_{L^{3}\left(\left(0, T_{0}\right) \times \Omega\right)}^{2}\right)
$$


(we recall that $W_{3}^{1,2}\left(\left(0, T_{0}\right) \times \Omega\right) \subset L^{\infty}\left(\left(0, T_{0}\right) \times \Omega\right)$ for $N \in\{1,2,3\}$; see $[1,8])$. So by (4.27) we have

$$
\left\|\tilde{p}^{\tilde{u}}\right\|_{L^{\infty}\left(\left(0, T_{0}\right) \times \Omega\right)}^{2} \leq C\left\|\tilde{p}_{\varepsilon}(0)\right\|_{L^{\infty}(\Omega)}^{2} .
$$

For $\left(u, p^{u}\right)$ given by $\left(\tilde{u}, \tilde{p}^{\tilde{u}}\right)$ on each characteristic line we have that $u \in$ $L^{2}\left(Q_{T}\right), p^{u}$ is the solution of (4.7) and $p(a, t, x)=0$ a.e. $(a, t, x) \in \Gamma_{0} \times \Omega$. Moreover,

$$
\left\|p^{u}\right\|_{L^{\infty}\left(Q_{2 T_{0}}\right)} \leq C\left\|\bar{p}_{0}\right\|_{L^{\infty}\left(\left(0, a_{\dagger}\right) \times \Omega\right)}+\|b\|_{L^{\infty}\left(\left(0,2 T_{0}\right) \times \Omega\right)} .
$$

We are now ready to prove our null exact controllability result. For any $b \in K$, we denote by $\Phi(b) \subset L^{2}\left(\left(0,2 T_{0}\right) \times \Omega\right)$ the set of all $\int_{0}^{a_{\dagger}} \beta(a) p^{u}(a, t, x) d a$, such that $u \in L^{2}\left(Q_{2 T_{0}}\right), p^{u}$ satisfies (4.32) and

$$
p^{u}(a, t, x)=0, \quad \text { a.e. }(a, t, x) \in \Gamma_{0} \times \Omega .
$$

There exists an element in $\Phi(b)$ which does not depend on $b$ :

- If $t>T_{0}$ then $\int_{0}^{a_{\dagger}} \beta(a) p^{u}(a, t, x) d a=0$. This is because $\beta(a)=0$ a.e. $a \in\left(0, T_{0}\right)$ and $p^{u}\left(a, T_{0}, x\right) d a=0$ for $a \geq T_{0}$.

- If $t \in\left(0, T_{0}\right)$ then $\int_{0}^{a_{\dagger}} \beta(a) p^{u}(a, t, x) d a=\int_{T_{0}}^{a_{\dagger}-T_{0}} \beta(a) p^{u}(a, t, x) d a$, and this depends only on $\bar{p}_{0}$ and not on $b$.

We also have that $p^{u}\left(a, 2 T_{0}, x\right)=0$ a.e. $(a, x) \in\left(0, a_{\dagger}\right) \times \Omega$ and

$$
\int_{T_{0}}^{a_{\dagger}-T_{0}} \beta(a) p^{u}(a, t, x) d a \leq C\|\beta\|_{L^{\infty}\left(0, a_{\dagger}\right)} \cdot\left\|\bar{p}_{0}\right\|_{L^{\infty}\left(\left(0, a_{\dagger}\right) \times \Omega\right)} .
$$

So, for any $u$ as above we can take

$$
b(t, x)= \begin{cases}0 & \text { a.e. }(t, x) \in\left(T_{0}, 2 T_{0}\right) \times \Omega, \\ \int_{0}^{a_{\dagger}} \beta(a) p^{u}(a, t, x) d a & \text { a.e. }(t, x) \in\left(0, T_{0}\right) \times \Omega\end{cases}
$$

a fixed point of the multivalued function $\Phi$. In addition by (4.32) and (4.34) we have

$$
\left\|p^{u}\right\|_{L^{\infty}\left(Q_{2 T_{0}}\right)} \leq C\left\|\bar{p}_{0}\right\|_{L^{\infty}\left(\left(0, a_{\dagger}\right) \times \Omega\right)} .
$$

So, if $\left\|\bar{p}_{0}\right\|_{L^{\infty}\left(\left(0, a_{\dagger}\right) \times \Omega\right)}$ is small enough, there exists $u \in L^{2}\left(Q_{2 T_{0}}\right)$ and $p$, the solution of (1.7) satisfies

$$
\begin{gathered}
p\left(a, 2 T_{0}, x\right)=0 \quad \text { a.e. }(a, x) \in\left(0, a_{\dagger}\right) \times \Omega, \\
\|p\|_{L^{\infty}\left(Q_{2 T_{0}}\right) \leq C\left\|\bar{p}_{0}\right\|_{L^{\infty}\left(\left(0, a_{\dagger}\right) \times \Omega\right)} \leq \rho_{0}}
\end{gathered}
$$

and in conclusion $p(a, t, x) \geq-\rho_{0}$ a.e. $(a, t, x) \in Q_{2 T_{0}}$. On the other hand, $p(a, t, x)$ does not depend on the control for $(a, t, x) \in\left(a_{\dagger}-T_{0}, a_{\dagger}\right) \times\left(0,2 T_{0}\right) \times$ $\Omega$, so

$$
p(a, t, x) \geq-p_{s}(a, x) \quad \text { a.e. in } Q_{2 T_{0}} .
$$


Now if we extend this $u$ by 0 outside $G \times \Omega$, we conclude the null controllability for (1.7) and the controllability for (1.1).

\section{References}

[1] R. A. Adams, Sobolev Spaces, Pure and Applied Mathematics, vol. 65, Academic Press, New York, 1975. MR 56\#9247.

[2] V. Barbu, Exact controllability of the superlinear heat equation, Appl. Math. Optim. 42 (2000), no. 1, 73-89. MR 2001i:93010. Zbl 0964.93046.

[3] E. Fernandez-Cara, Null controllability of the semilinear heat equation, ESAIM Control Optim. Calc. Var. 2 (1997), 87-103. MR 98d:93011. Zbl 0897.93011.

[4] A. V. Fursikov and O. Yu. Imanuvilov, Controllability of Evolution Equations, Lecture Notes Series, vol. 34, Seoul National University, Seoul, 1996. MR 97g:93002. Zbl 0862.49004.

[5] M. G. Garroni and M. Langlais, Age-dependent population diffusion with external constraint, J. Math. Biol. 14 (1982), no. 1, 77-94. MR 84i:92069. Zbl 0506.92018.

[6] M. E. Gurtin, A system of equations for age dependent population diffusion, J. Theor. Biol. 40 (1972), 389-392.

[7] M. Iannelli, Mathematical Theory of Age-Structured Population Dynamics, Applied Mathematics Monograph, vol. 7, Giardini Editori e Stampatori, Pisa, 1995.

[8] O. A. Ladyženskaja, V. A. Solonnikov, and N. N. Ural'ceva, Linear and Quasilinear Equations of Parabolic Type, Mathematical Monographs, vol. 23, American Mathematical Society, Rhode Island, 1967. MR 39\#3159b.

[9] M. Langlais, Large time behavior in a nonlinear age-dependent population dynamics problem with spatial diffusion, J. Math. Biol. 26 (1988), no. 3, 319-346. MR 89f:92046. Zbl 0713.92019.

[10] G. Lebeau and L. Robbiano, Contrôle exact de l'équation de la chaleur [Exact control of the heat equation], Comm. Partial Differential Equations 20 (1995), no. 1-2, 335-356 (French). MR 95m:93045.

[11] G. F. Webb, Theory of Nonlinear Age-Dependent Population Dynamics, Monographs and Textbooks in Pure and Applied Mathematics, vol. 89, Marcel Dekker, New York, 1985. MR 86e:92032. Zbl 0555.92014.

Bedr'Eddine Ainseba: Université Victor Segalen Bordeaux II, 33076 Bordeaux Cedex, France et Mathématiques appliquées de Bordeaux, UMR CNRS 5466, France

E-mail address: ainseba@sm.u-bordeaux2.fr

Sebastian Aniţa: Faculty of Mathematics, University "Al.I. CuZA" and Institute of Mathematics of the Romanian Academy, Iaşi 6600, Romania

E-mail address: sanita@uaic.ro 


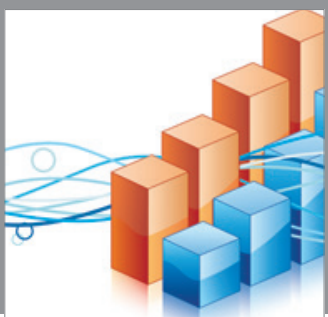

Advances in

Operations Research

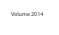

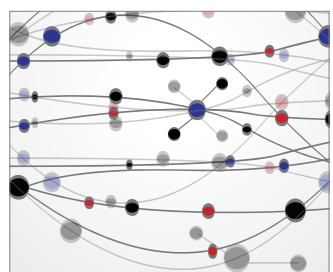

\section{The Scientific} World Journal
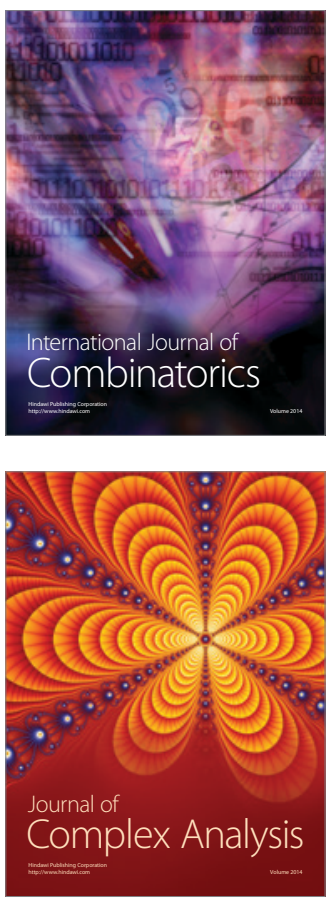

International Journal of

Mathematics and

Mathematical

Sciences
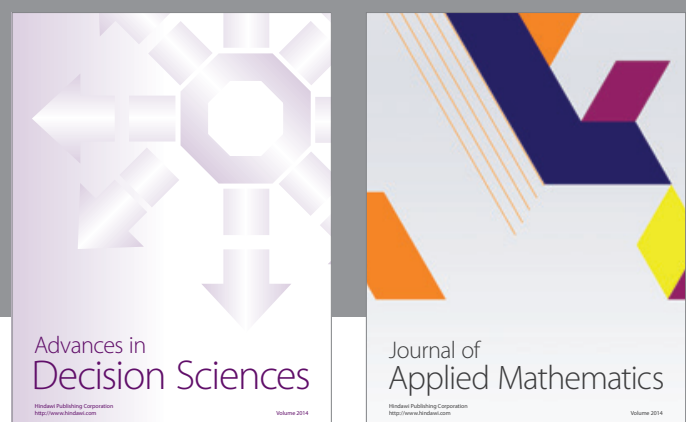

Journal of

Applied Mathematics
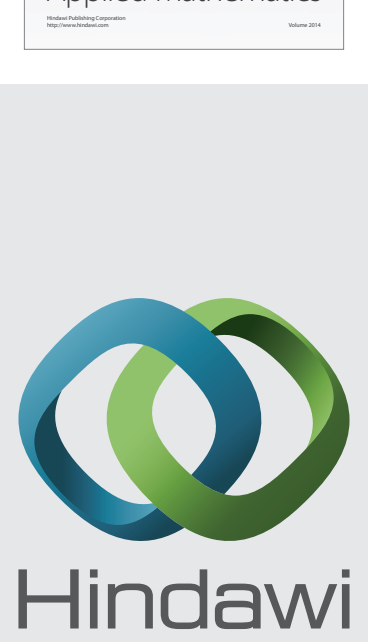

Submit your manuscripts at http://www.hindawi.com
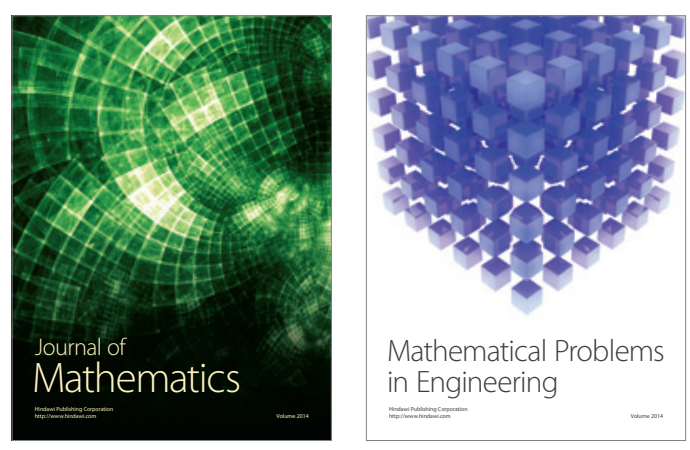

Mathematical Problems in Engineering
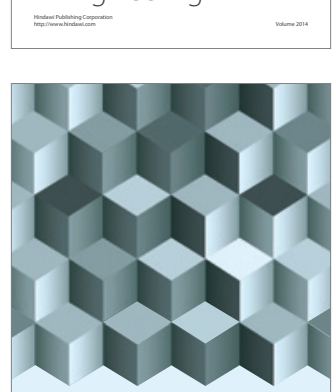

Journal of

Function Spaces
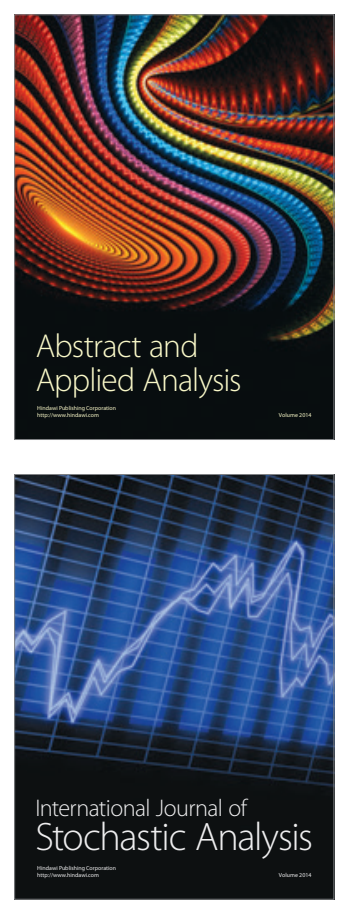

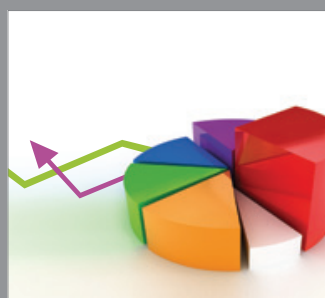

ournal of

Probability and Statistics

Promensencen
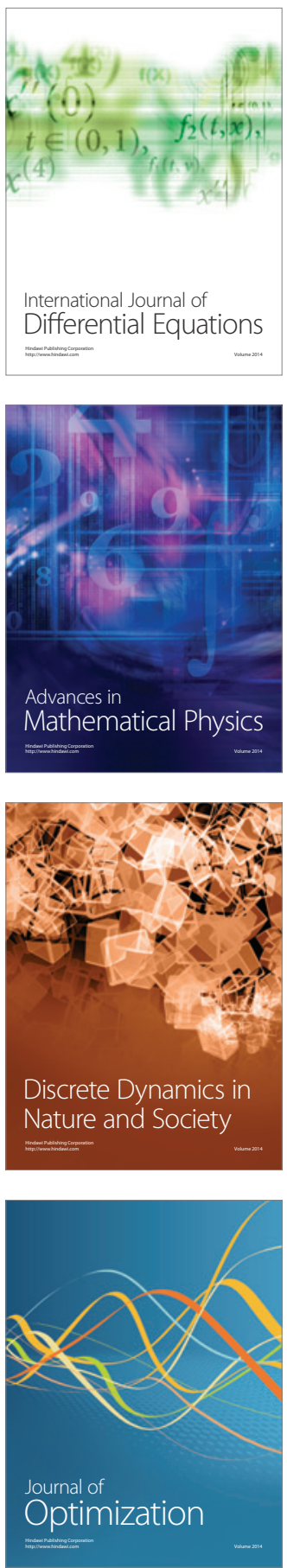